\title{
Upconverter Nanoparticles as Plasmon-induced Broadband Light Converters
}

\author{
Vallee Renaud Arthur Leon \\ Paul Pascal Research Center, University of Bordeaux, The French National Centre for Scientific Research, Pessac, France
}

\author{
Email address: \\ renaud.vallee@crpp.cnrs.fr
}

\section{To cite this article:}

Vallee Renaud Arthur Leon. Upconverter Nanoparticles as Plasmon-induced Broadband Light Converters. American Journal of Nanosciences. Vol. 7, No. 2, 2021, pp. 35-41. doi: 10.11648/j.ajn.20210702.11

Received: April 9, 2021; Accepted: April 22, 2021; Published: May 8, 2021

\begin{abstract}
We show numerically how lanthanide-doped upconverter nanocrystals inserted at optimal positions in adjustable and smooth 2D plasmonic arrays may change and potentially control the whole UV-visible-NIR reflectance spectra displayed by these structures. The analysis and interplay between a custom-built simple 5-levels 2-electrons quantum model and the bare structure have been studied in depth and revealed very fundamental and interesting physics. Essentially, the largely and selectively enhanced white-light excitation field and optimized local density of states at the location of the emitters favor some energy transfer paths and a redistribution of light energy in a broad spectral range. Interestingly, the extent to which the spectra can be modulated owing to the emission properties of such very robust multilevel emitters may find interesting applications and notably allow increased efficiency of emission in Light Emitting Devices or solar light redistribution and collection in Solar cells, where conversions from one color to any other one play a major role.
\end{abstract}

Keywords: Plasmonic Array, Gap Mode Resonance, Upconverters, FDTD

\section{Introduction}

Photon upconversion in lanthanide-doped nanoparticles (UCNPs) involves multiple mechanisms of electronic transitions [1]. Stepwise optical excitation, energy transfer, and various nonlinear and collective light-matter interaction processes act together to convert low-energy photons into high-energy or short-wavelength light emission [1]. At the nanoscale, the occurrence and efficiency of such anti- Stokes emission crucially depends on the size, surface modification and functionalization, co-doping with semiconductor nanocrystals or other lanthanides, core-shell processing of the crystals and controlled nanocrystal assembly [2-6]. Magnetic and electric field-induced tuning, thermal phonon activation, mechanical stress-induced tuning, anisotropic polarization modulation, altering the $\mathrm{pH}$ environment and photonic crystal engineering are other routes which have been followed ingeniously with some important successes, to tune the band gap energy levels and optimize the up-conversion emission [7-13]. Coupling UCNPs with surface plasmons has been recently quoted as one of the most promising strategies to apply as a lever to enhance one or the other or both of the
UCNPs' excitation and emission rates [14-19]. The targeted applications are broad and cover, non-exhaustingly, the fields of solid-state lasing, IR imaging, security printing, volumetric displays, photovoltaic devices, up to biological imaging and therapeutics [13, 20, 21]. The emerging application of an ultralow-threshold upconverting laser operating at room temperature [20] could be designed owing to a careful engineering of a 2D lattice exhibiting a sharp lattice resonance coupled to the sharp red emission line of a film of $14 \mathrm{~nm}$ core-shell UCNPs $\left(\mathrm{NaYF}_{4}: \mathrm{Yb}^{3+}, \mathrm{Er}^{3+}\right)$.

In the current paper, we aim to go one step further and, based on a very recently published paper [22], show numerically how lanthanide-doped upconverter nanocrystals inserted at optimal positions in adjustable and smooth 2D plasmonic arrays may benefit from the largely and selectively enhanced white-light excitation field and optimized local density of states (Purcell effect [23]) to favor some energy transfer paths and thus redistribute light energy in a broad spectral range, as tracked by reflectance spectra. Such results open new avenues towards the realization of new, optimized, types of solar cells based on array plasmon-induced photon upconversion $[21,24]$. 


\section{Methods}

The numerical Finite Difference Time Domain (FDTD) simulations have been performed with Lumerical [28]. The structure has been designed in a way to mimic as closely as possible the real structure. Accordingly, in a first step, a 150 $\mathrm{nm}$ gold layer has been deposited on a glass substrate layer, followed by the extrusion of perpendicular semi- ellipsoidal rows with adequate depths and lateral size $(25 \mathrm{~nm})$, featuring the nanotrenches (gaps) periodically reproduced in a lattice. The lattice constants of the structure have been set to 60,120 , $180,240,300,360 \mathrm{~nm}$ for gap depths of 50 to 52.5, 60, 67.5, 70 and $80 \mathrm{~nm}$, respectively. Anti- symmetric (symmetric) boundary conditions have been set parallel (perpendicular) to the excitation polarization direction (along X) while Perfectly Matched Layers were implemented in the Z-direction of a plane wave propagating backward while launched from the top of the structure. For the bare nanotrenches' 2D arrays, the reflectance spectra were then acquired in a Z-perpendicular plane well above (in order to avoid near field effects) the structure.

In a second step, in order to calculate the Purcell effect acting on the UCNPs due to the presence of the gap-mode resonance of the bare structure, we inserted a dipole (featuring the emitter) oriented transversally along $\mathrm{X}$ at a central hotpot position (between two perpendicular rows) and calculated its total radiated power. For each structure and considered wavelength, the obtained value was then normalized to the one of the same emitter placed in vacuum.

Finally, in order to investigate the interaction between UCNPs and the plasmonic structure, we used the Flexible material plugin framework of Lumerical in order to implement our simplified 5-levels 2- electrons custom-built quantum model. In all such simulations, the light intensity was kept very low, in order to avoid saturation effects.

In all simulations, an auto non-uniform mesh type with accuracy 6 has been used and the (Lumerical) Conformal Mesh Technology (CMT) was chosen, (the so-called conformal variant 0 ). To increase the accuracy and insure convergence, a sub-mesh with a step size of $2 \mathrm{~nm}$ was added in and immediately around the gaps (nanotrenches). The optical constants of glass $\left(\mathrm{SiO}_{2}\right)$ and gold $(\mathrm{Au})$ are the ones provided by Palik and Johnson and Christy, respectively.

\section{Results and Discussion}

Template stripping methods have been recently used to produce large-scale metallic structures with nanoscale precision and atomically smooth top surface $[25,26]$. A spreadmediated self-assembly method also permits the precise positioning of UCNPs into vertical nanoscale gaps created between the metal walls [27]. This situation is extremely profitable since these locations show the largest field enhancements. In a recent paper [22], the authors made use of their recently established platform, the template-stripped gold nanotrench, to confine UCNPs within nanoscale gaps between gold trenches. To achieve a precise positioning of
UCNPs, the gold nanotrenches' lateral sizes were reduced to as less as a sub- $25 \mathrm{~nm}$ scale to be able to accomodate a single row of UCNPs. Figure 1a here below shows a top view of the scheme for the $2 \mathrm{~d}$ nanotrench array they could typically obtain. The smoothness of the achieved structure [22] is essential in order to avoid huge scattering losses and inhibit the creation of rough sites-induced hotspots.

In the published paper [22], the authors show in Figure 2c the experimentally obtained spectra for various choices of the period P. For P varying from 60 to $360 \mathrm{~nm}$, the reflectance spectra encompass a large dip shift ranging from about 500 $\mathrm{nm}$ to more than $1000 \mathrm{~nm}$. The dip shift is further accompanied by a significant broadening of the dip.

In the current paper, we aim to provide evidence that the observation of such a large and profound displacement of the reflectance dip is not only the consequence of varying the constant $\mathrm{P}$ of the lattice, but also the depth $\mathrm{D}$ of the nanotrenches and, more importantly, the consequence of selective energy transfer and complete redistribution of light energy owing to excitonic interactions between UCNPs and gap-mode resonances when excited with white light illumination.

In order to demonstrate this, we firstly investigated, owing to numerical FDTD simulations [28], the mode profiles of various structures and adjusted the spectral locations of the respective mode resonances.

The fundamental mode of a plasmonic cavity is defined by the gap width, pitch size and vertical elongation of the structure, as well as the dielectric medium surrounding the metallic resonator. As shown in Figure 1b, the resonant mode is strongly localized in the gap region with a maximum field intensity enhancement $\left(\left|E / E_{0}\right|^{2}\right)$ reaching 280 for a structure with a $360 \mathrm{~nm}$ lattice constant. Such results also show that the incorporation of the UCNPs with a refractive index $n=1.5$ into the nanogap further concentrates the fields in two electromagnetic field intensity hotspots located between the particles and trench side-walls. Such a situation is likely to favor a deep interplay between both elements.

We thus firstly simulated 6 structures in the absence of UCNPs, with lattice constants P increasing from 60 to 120 , 180, 240, 300 and $360 \mathrm{~nm}$ while nanotrenches' depths D correspondingly increase from 50 to $52.5,60,67.5,70$ and 80 $\mathrm{nm}$, keeping the gap width $\mathrm{S}$ to $20 \mathrm{~nm}$ in order to accommodate a single row of UCNPs.

Figure $2 \mathrm{a}$ shows the reflectance spectra for such structures, exhibiting dips (resonances) ranging from about $540 \mathrm{~nm}$ to $800 \mathrm{~nm}$. Such a nice red shift of the reflectance dip as a function of the period and depth of the nanotrenches in the $2 \mathrm{D}$ array is in fact due to the interaction between a photonic mode whose properties depend on the periodicity $\mathrm{P}$ of the structure and a plasmonic mode for which the shape and, in particular, the aspect ratio $\mathrm{D} / \mathrm{S}$ of the nanotrenches govern the sloshing of the electrons in the trench side walls. It has to be noted here that uniquely varying the period of the structure does induce a red-shift of the resonance mode, but to a much lesser extent than modulating as well the aspect ratio of the nanotrenches. Also, while the red shift shows up already 
nicely, it is far from reaching the amplitude of the one experimentally observed in Figure 2c of the reference paper [22], in the presence of UCNPs.

Our next step concerns the true target of this paper: investigating how the insertion of a large number of upconverter nanoparticles in the rows of the nanotrenches' struc- ture (Figure 1a) may lead to a redistribution of light energy owing to the fact that the UCNPs benefit from both an exalted localized excitation field and an increased emission originating from an increased local density of states (LDOS, Purcell effect [23]) provided by the metallic elements, at the location of their immobilization (Figure 1b).

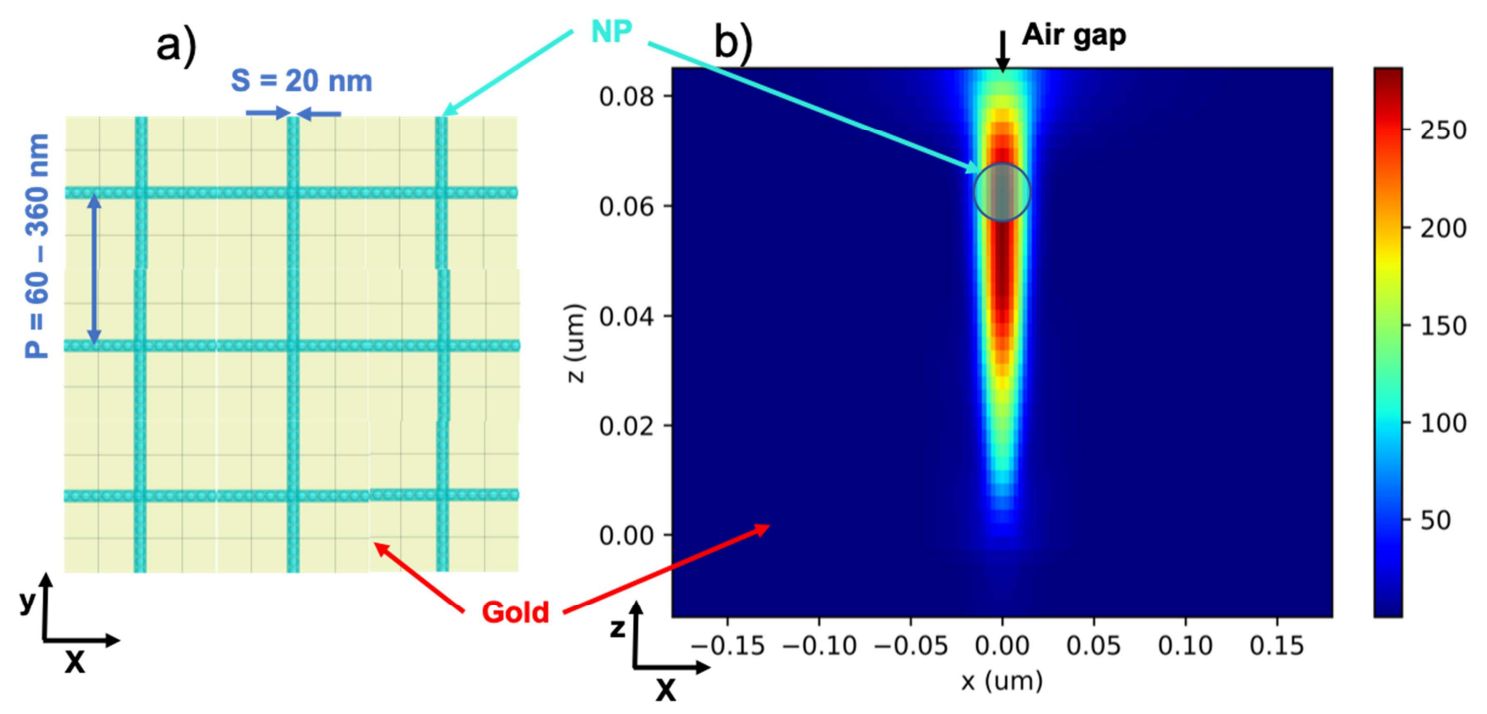

Figure 1. a) Top view (scheme) of a UCNPs-gold plasmonic hybrid structure: UCNPs are inserted in periodic (period P varying between 60 and 360 nm) nanotrenches in gold of sub $25 \mathrm{~nm}$ width $S$. b) Lateral view of the FDTD-simulated gap mode for a $360 \mathrm{~nm}$ periodic array.

UCNPs possess a large amount of energy states, with many allowed transitions potentially occurring between them [1]. For the $\mathrm{NaYF}_{4}: \mathrm{Yb}^{3+}, \mathrm{Er}^{3+}$ UCNPs here selected, it is well-known that the dominant anti-Stokes emission paths

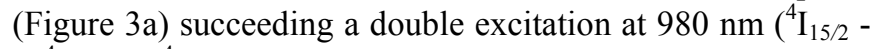
$\left.>{ }^{4} \mathrm{I}_{11 / 2} \rightarrow{ }^{4} \mathrm{~F}_{7 / 2}\right)$ are the green emission occurring at $540 \mathrm{~nm}$ $\left({ }^{4} \mathrm{~S}_{3 / 2}->{ }^{4} \mathrm{I}_{15 / 2}\right)$ and the red emission occurring at $655 \mathrm{~nm}\left({ }^{4} \mathrm{~F}_{9 / 2}\right.$ $\left.\rightarrow{ }^{4} \mathrm{I}_{15 / 2}\right)$ [18]. We want here to investigate the response of such upconverter nanoparticles precisely located in our plasmonic nanostructures when excited via white light

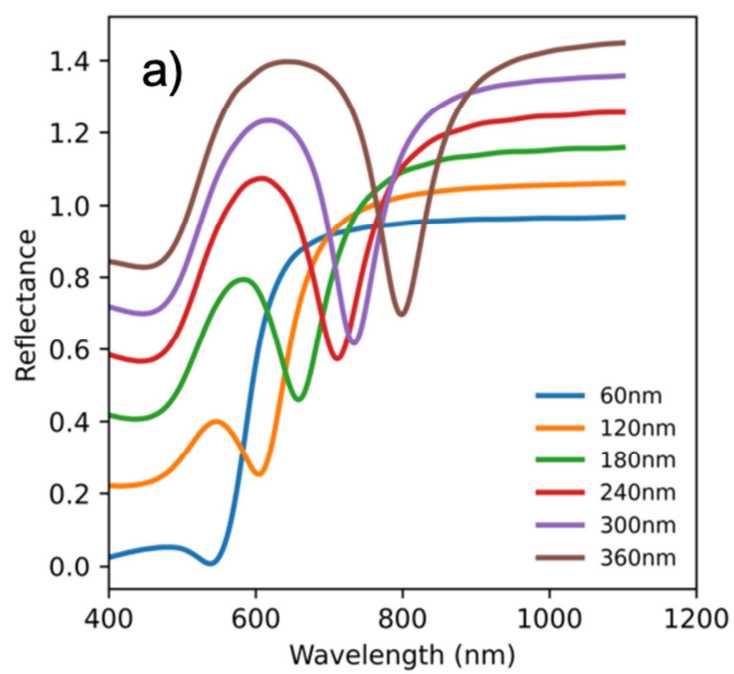

illumination (Figure 2b) instead of a usual single monochromatic light at the $980 \mathrm{~nm}$ excitation wavelength [24]. The aim is to elucidate the role of the various excitation and emission pathways, operating with a strength depending on their spectral overlap with the gap-mode resonances of the used structures, on the energy redistribution in the system.

Such a study is eminently complex if one wants to keep too many parameters in the description. Accordingly, we chose here to set up a custom-built, very simplified 5-levels 2-electrons, quantum model of the UCNPs (Figure 3a).

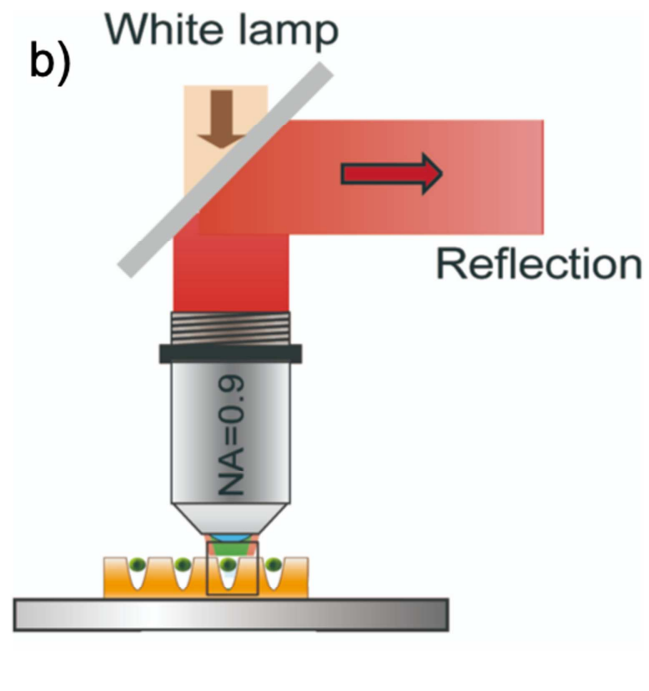

Figure 2. a) Reflectance spectra of gold nanotrenches' $2 \mathrm{D}$ arrays with different lattice constants (60-360 $\mathrm{nm}$ ) and depths of the nanotrenches (50-80 nm) obtained in the case of numerical FDTD simulations; b) white light illumination and spectrographically resolved detection as performed in the reference paper [22]. 

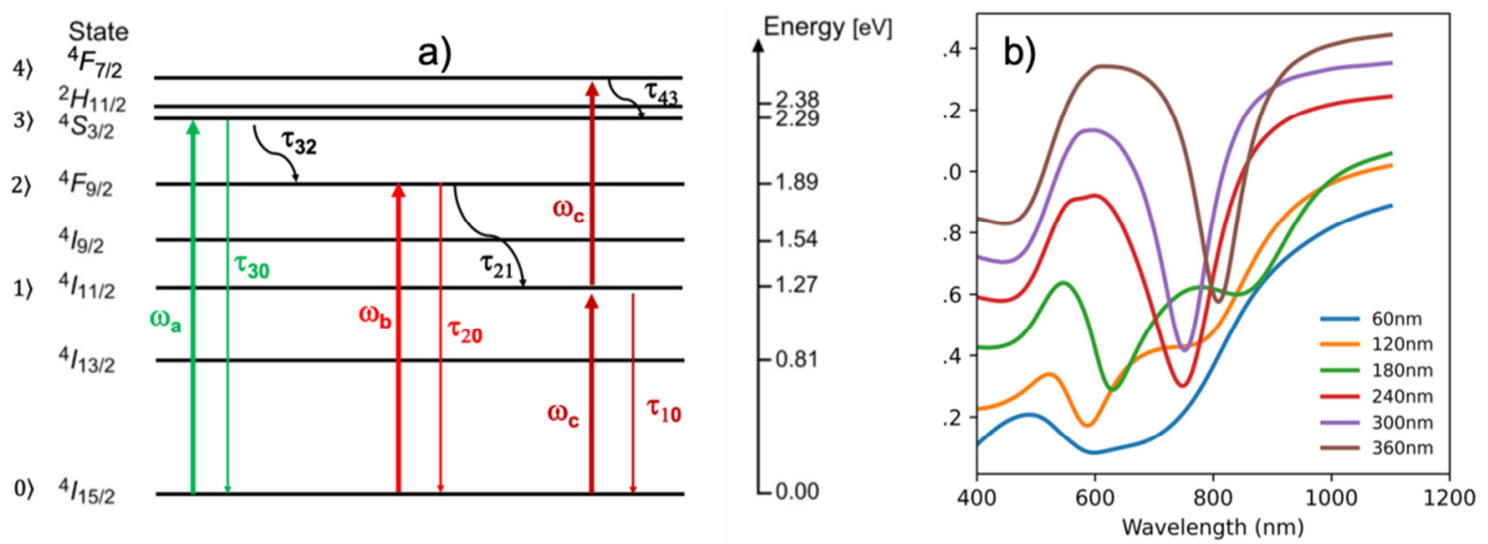

Figure 3. a) Simplified 5 levels - 2 electrons model of Er ${ }^{3+}$ ions; b) Numerically obtained UV-visible-NIR spectra as upconverter nanoparticles are inserted in the structures.

Of course, the electronic structure of $\mathrm{Er}^{3+}$ ions involves many more levels and relaxation paths between them [6] However, we want to specifically focus on the two main transitions occurring in the visible range of the spectrum, e.g. the ${ }^{4} \mathrm{~S}_{3 / 2} \rightarrow{ }^{4} \mathrm{I}_{15 / 2}$ and ${ }^{4} \mathrm{~F}_{9 / 2} \rightarrow{ }^{4} \mathrm{I}_{15 / 2}$ transitions. As we chose to employ a white light illumination, all energy levels can a priori be excited. Nevertheless, as observed in the reflectance spectra of the structures without UCNPs (Figure 2a), the resonance modes are likely to essentially act on the two above mentioned transitions, so that we will mainly consider (plasmonically enhanced) excitation of these two levels. We also allow the excitation of the ${ }^{4} \mathrm{I}_{11 / 2}$ and ${ }^{4} \mathrm{~F}_{7 / 2}$ through the traditional GSA-ESA processes at a wavelength of $980 \mathrm{~nm}$ as the lowest order upconversion mechanism potentially playing a role (Figure 3a).

As such, the decay rate equations of the gain medium, describing the multiphoton pump and emission processes are:

$$
\begin{gathered}
\frac{d N_{4}}{d t}=-\frac{1}{\tau_{43}} N_{4}\left(1-N_{3}\right)+\frac{1}{\hbar \omega_{c}} \mathrm{E} \frac{d P_{c}}{d t} \frac{N_{1}}{N_{0}+N_{1}} \\
\frac{d N_{3}}{d t}=-\frac{1}{\tau_{30}} N_{3}\left(1-N_{0}\right)-\frac{1}{\tau_{32}} N_{3}\left(1-N_{2}\right)+\frac{1}{\tau_{43}} N_{4}\left(1-N_{3}\right)+\frac{1}{\hbar \omega_{a}} \mathrm{E} \frac{d P_{a}}{d t} \\
\frac{d N_{2}}{d t}=-\frac{1}{\tau_{20}} N_{2}\left(1-N_{0}\right)-\frac{1}{\tau_{21}} N_{2}\left(1-N_{1}\right)+\frac{1}{\tau_{32}} N_{3}\left(1-N_{2}\right)+\frac{1}{\hbar \omega_{b}} \mathrm{E} \frac{d P_{b}}{d t} \\
\frac{d N_{1}}{d t}=-\frac{1}{\tau_{10}} N_{1}\left(1-N_{0}\right)+\frac{1}{\tau_{21}} N_{2}\left(1-N_{1}\right)+\frac{1}{\hbar \omega_{c}} \mathrm{E} \frac{d P_{c}}{d t} \frac{N_{0}-N_{1}}{N_{0}+N_{1}} \\
\frac{d N_{0}}{d t}=-\sum_{i=1}^{4} \frac{d N_{i}}{d t}
\end{gathered}
$$

with $\mathrm{N}_{0}, \ldots, \mathrm{N}_{4}$ the normalized population densities of the various levels, $\hbar$ the reduced Planck constant, $\omega_{a}, \omega_{b}, \omega_{c}$ the pump frequencies and $\mathrm{P}_{a}, \mathrm{P}_{b}, \mathrm{P}_{c}$ the various absorption and emission polarization fields (Figure 3a). For the sake of our numerical simulations, we chose a realistic value of the population density of the whole system equal to $1.5 * 10^{26} / \mathrm{m}^{3}$. The transition lifetimes are $\tau_{43}=\tau_{32}=\tau_{21}=10 \mathrm{fs}$, chosen to be so short since they correspond to the fastly decaying channels of multi phonon relaxation (MPR). $\tau_{30}, \tau_{20}$ and $\tau_{10}$ are equal to 10, 19 and $95 \mathrm{~ns}$, respectively, with ratios provided by the Judd-Ofelt analysis of the Einstein coeffi- cients performed in previous study [29] for the spontaneous emission of the $\beta$ $\mathrm{NaEr} 0.2 \mathrm{Y}_{0.8} \mathrm{~F}_{4}$. Compared to the transition lifetimes of $\mathrm{Yb}$ /Er -co-doped UCNPs reported in this study, the values are reduced to allow for simulation within the computational window $(<1 \mathrm{ps})$ in the time-domain approach.

In order to perform the analysis, these rate equations are of course combined to the oscillator equations that describe the induced population difference driven by the absorption and emission polarization fields. In this model, four electronic transitions are triggered under optical pump at 540, 655 and
$980 \mathrm{~nm}$, while being at the same time coupled to gap-mode

$$
\begin{gathered}
\frac{d^{2} P_{j}}{d t}+\gamma_{j} \frac{d P_{j}}{d t}+\omega_{j}^{2} P_{j}=\xi_{j} E\left(N_{f}-N_{i}\right) \\
\xi_{j}=\frac{6 \pi c^{3}}{\omega_{j}^{2} \tau_{f i}}
\end{gathered}
$$

with $\mathrm{j}$ belonging to $\{\mathrm{a}, \mathrm{b}, \mathrm{c}\}$ levels and (i, f) belonging to $\{(0,3),(0,2),(0,1)\}$ transitions (Figure 3a). Once inserted in the nanotrenches' 2D arrays, UCNPs interact with the gapmode resonance and benefit from the enhanced local electric field E. In such a situation, UCNPs also act as emitters and are subjected to the Purcell effect induced by this mode. The emission rate of UCNPs in the various structures will thus be enhanced by a Purcel factor $F_{p}(\lambda, T)$, which is a function of the wavelength, polarization and location of the emitter in the considered structure, this factor varying also with the structure periodicity. Table 1 collects such Purcell factors calculated for a dipole emitter polarized along $\mathrm{X}$ and located at the intersection of two perpendicular (along $X$ and along Y) nanotrenches at a depth of $15 \mathrm{~nm}$. Clearly, the Purcell 
factor is significant, with values from a few tens to a few hundreds at the wavelengths of interest, depending on the period of the considered structure.

Table 1. Purcell factors $F_{p}(\lambda, T)$ for specific emission wavelengths and various lattice constants of the structure.

\begin{tabular}{llll}
\hline \multirow{2}{*}{ Lattice constant (nm) } & \multicolumn{2}{l}{ Wavelenth $(\mathbf{n m})$} \\
\cline { 2 - 4 } & $\mathbf{5 4 0}$ & $\mathbf{6 5 5}$ & $\mathbf{9 8 0}$ \\
\hline 60 & 176 & 127 & 58 \\
120 & 88 & 180 & 31 \\
180 & 88 & 215 & 26 \\
240 & 106 & 71 & 25 \\
300 & 92 & 41 & 23 \\
360 & 65 & 28 & 32 \\
\hline
\end{tabular}

According to these Purcell factor values, the $\tau_{30}, \tau_{20}$ and $\tau_{10}$ decay times of the UCNPs are thus rescaled as

$$
\tau_{i 0}=\frac{\tau_{i 0}}{F_{\rho}(\lambda, T)}
$$

in all FDTD simulations, for the sake of taking fully into account the fact that there are inserted at well-given positions and thus benefit from the increased LDOS at these specific locations in the structure. The $\tau_{43}=\tau_{32}=\tau_{21}$ decay rates, describing MPR, are unaffected.

As shown in Figure 3b, and very interestingly, the UVvisible spectra are strongly affected by the presence of the emitters located at hot spots in the structure. As such, one sees, by comparison with Figure $2 \mathrm{a}$, an overall red shift and broadening of the reflectance dips for all structures, with a secondary dip appearing slightly below and above a wavelength of $800 \mathrm{~nm}$ in the $120 \mathrm{~nm}$ and $180 \mathrm{~nm}$ periodic structures, respectively. Also, more broadly expanded maximal peaks occur on the blue side of the reflectance dip, for periodic structures with a long period.

As such, on assists indeed to a redistribution of light energy, as due to the presence of UCNPs particles excited via a white light lamp (Figure 2b). The observed shifts are nevertheless not in good agreement with the ones experimentally observed in Figure 2c of the reference paper [22], especially for the periodic structures with larger lattice constants, where the resonance modes are much more red-shifted.

Obviously, some energy levels might lack in our model Equations (1-7) or some parameters might have been inadequately set, which would explain the discrepancy. By comparing the experimental reflectance spectra of the structures with UCNPs (Figure 2c of the reference paper [22]) to the ones without UCNPs here simulated (Figure 2a), one could argue that UCNPs particles modify the reflectance spectra and thus redistribute light energy by emitting strongly in the green spectral region while not so much in the red band. Accordingly, while the measured reflectance would increase due to the increased emission of the green band, the dip would appear in the red region as an effect of absorption of the excitation light in the red part of the spectrum, not followed by emission at these wavelengths.

In agreement with these considerations, we envisage a new situation where, instead of rescaling the $\tau_{20}$ and $\tau_{10}$ decay times with the calculated Purcell factors $F_{p}(\lambda, T)$ (Equation
(8)), one blocks, on an appropriate time scale, the radiative emission at $655 \mathrm{~nm}$ as well as the one at $980 \mathrm{~nm}$ while favoring the green emission by shortening its relaxation time $\tau_{30}$ even further than previously performed. By blocking the red emission, the ${ }^{4} \mathrm{~F}_{9 / 2}$ level could then only decay through $\operatorname{MPR}\left(\mathrm{N}_{2}->\mathrm{N}_{1}\right)$ towards the ${ }^{4} \mathrm{I}_{11 / 2}$ level. The decay from this level being blocked as well, the pump at $980 \mathrm{~nm}$ acting on this level would populate $\left(\mathrm{N}_{1}->\mathrm{N}_{4}\right)$ the ${ }^{4} \mathrm{~F}_{7 / 2}$ level, which, in turn would depopulate $\left(\mathrm{N}_{4}->\mathrm{N}_{3}\right)$ via MPR to the ${ }^{4} \mathrm{~S}_{3 / 2}$ level, whose emission would finally be strongly reinforced. On a reflectance spectrum, such effect would appear as a decrease (dip) in the red $(655 \mathrm{~nm})$ and near-IR $(980 \mathrm{~nm})$ spectral regions concomitant with a boost at shorter wavelength, corresponding to the green spectral region.

According to this reasoning, we performed a new set of FDTD simulations, for all structures, setting $\tau_{20}=\tau_{10}=10 \mathrm{~ns}$ and adjusting $\tau_{30}$ at best $\left(\tau_{30}=113.8,114.0,56.8,18.8,18.2\right.$, $15.4 \mathrm{ps}$ for the $60,120,180,240,300,360 \mathrm{~nm}$ periodic structures, respectively). The new numerically obtained reflectance spectra are shown in Figure 4a. Clearly, these spectra actually compare much better in a qualitative, if still not on a completely quantitative, way with the experimental spectra (Figure 2c of reference [22]). The major dip is clearly pushed to the red side of the spectrum as the lattice constant (and associated trenches' depth) of the structure is increased. The secondary dip, on the blue side, experimentally often barely recognizable, is now also clearly observable in the spectra.

Very interestingly, such nice spectral features surge together with a very fast increase of population / relaxation steps of the ${ }^{4} \mathrm{~S}_{3 / 2}$ (green) level, taking place much earlier than the population of the ${ }^{4} \mathrm{~F}_{9 / 2}$ (red) level (Figure $4 \mathrm{c}$ ), as anticipated owing to the arguments we put forwards at the initial stage of this set of simulations, as compared to the previous set (Figure $4 \mathrm{~b}$ ). It is interesting to note how such a simplified 5-levels 2-electrons quantum model of UCNPs interacting with the gap-mode resonances offers such a good and already semi-quantitative insight on the way to modulate spectral features of the hybrid structure.
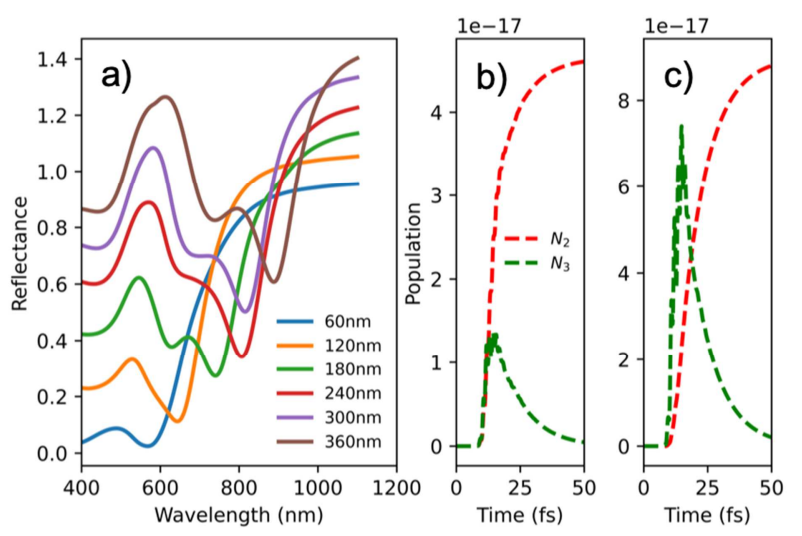

Figure 4. FDTD numerically calculated reflectance spectra of the UCNPScoupled nanotrenches' $2 D$ arrays (a) and populations of the ${ }^{4} S_{3 / 2}\left(N_{3}\right)$ and ${ }^{4} F_{9 / 2}\left(N_{2}\right)$ energy levels as a function of time after pumping, for a structure with a $360 \mathrm{~nm}$ lattice constant and parameters matching the situation leading to Figure $2 b$ (b) and Figure $4 a$ (c). 


\section{Conclusions}

In conclusion, we showed here how to design and engi- neer smooth plasmonic nanotrenches' 2D arrays accomodat- ing UCNPs at carefully chosen hotpots in order to change and potentially control the whole UV-visible-NIR reflectance spectra displayed by these structures. The analysis and interplay between a custom-built simple 5-levels 2-electrons quantum model and the bare structure have been studied in depth and revealed very fundamental and interesting physics. The real experimental realization semi-quantitatively confirmed and allowed to optimize the model. Interestingly, the extent to which the spectra can be modulated owing to the emission properties of such very robust multilevel emitters may find interesting applications and notably allow in- creased efficiency of emission in Light Emitting Devices or solar light redistribution and collection in Solar cells, where conversions from one color to any other one play a major role.

\section{References}

[1] Guokui Liu "Advances in the theoretical understanding of photon upconversion in rare- earth activated nanophosphors". Chemical Society Reviews, 44 (6): 1635-1652, 2015.

[2] F Zhang "Mesoporous multifunctional upconversion luminescent and magnetic "nanorattle" materials for targeted chemotherapy". Nano Letters, 12: 61-67, 2012.

[3] Le-Le Li, Peiwen Wu, Kevin Hwang, and Yi Lu "An Exceptionally Simple Strategy for DNA- Functionalized UpConversion Nanoparticles as Biocompatible Agents for Nanoassembly, DNA Deliv- ery, and Imaging". Journal of the American Chemical Society, 135 (7): 2411-2414, 2013.

[4] Z Li, L Wang, Z Wang, X Liu, and Y Xiong "Modification of NaYF 4: Yb, Er@SiO2 nanoparticles with gold nanocrystals for tunable green-to-red upconver- sion emissions". J. Phys. Chem. C, 115: 3291-3296, 2011.

[5] X Wang, X Kong, Y Yu, Y Sun, and H Zhang "Effect of annealing on upconversion luminescence of $\mathrm{ZnO}$ : $\mathrm{Er} 3+$ nanocrystals and high thermal sensitivity". J. Phys. Chem. C, 111: 15119-15124, 2007.

[6] Bo Zhou, Bingyang Shi, Dayong Jin, and Xiaogang Liu "Controlling upconversion nanocrystals for emerging applications". Na- ture Nanotechnology, 10 (11): 924-936, 2015.

[7] Jiajia Zhou, Shihui Wen, Jiayan Liao, Christian Clarke, Sherif Abdulkader Tawfik, Wei Ren, Chao Mi, Fan Wang, and Dayong Jin "Activation of the surface dark- layer to enhance upconversion in a thermal field". Nature Photonics, 12 (3): 154-158, 2018.

[8] Z Yin, Y Zhu, W Xu, J Wang, S Xu, B Dong, L Xu, S Zhang, and $\mathrm{H}$ Song "Remarkable enhancement of upconversion fluorescence and confocal imaging of PMMA Opal/NaYF 4: Yb3+, Tm3+ / Er3+ nanocrystals". Chem. Commun, 49: 3781-3784, 2013.

[9] J Hao, Y Zhang, and X Wei "Electric-induced enhance- ment and modulation of upconversion photolumines- cence in epitaxial BaTiO 3: $\mathrm{Yb} / \mathrm{Er}$ thin films". Angew. Chem. Int. Ed, 50: 6876-80, 2011.
[10] Jiajia Zhou, Gengxu Chen, E Wu, Gang Bi, Botao Wu, Yu Teng, Shifeng Zhou, and Jianrong Qiu "Ultrasensitive Polarized Up-Conversion of $\mathrm{Tm} 3+-\mathrm{Yb} 3+$ Doped $\beta$-NaYF4 Single Nanorod". Nano Letters, 13 (5): 2241-2246, 2013.

[11] Michael D. Wisser, Maverick Chea, Yu Lin, Di M. Wu, Wendy L. Mao, Alberto Salleo, and Jennifer A. Dionne "Strain-Induced Modification of Optical Selection Rules in Lanthanide-Based Upconverting Nanoparticles". Nano Letters, 15 (3): 1891-1897, 2015.

[12] Nicoleta Bogdan, Fiorenzo Vetrone, Geoffrey A. Ozin, and John A. Capobianco "Synthesis of Ligand-Free Colloidally Stable Water Dispersible Brightly Luminescent LanthanideDoped Upconverting Nanoparticles". Nano Letters, 11 (2): 835-840, 2011.

[13] Yiming Wu, Melgious Jin Yan Ang, Mingzi Sun, Bo- long Huang, and Xiaogang Liu "Expanding the tool- box for lanthanide-doped upconversion nanocrystals". Journal of Physics D: Applied Physics, 52 (38): 383002, 2019.

[14] Wen $\mathrm{Xu}, \mathrm{Xu}$ Chen, and Hongwei Song "Upconversion manipulation by local electromagnetic field". Nano Today, 17: 54-78, 2017.

[15] Wounjhang Park, Dawei Lu, and Sungmo Ahn "Plasmon enhancement of luminescence upconversion". Chemical Society Reviews, 44 (10): 2940-2962, 2015.

[16] Jun Dong, Wei Gao, Qingyan Han, Yongkai Wang, Jianxia Qi, Xuewen Yan, and Mengtao Sun "Plasmon- enhanced upconversion photoluminescence: Mechanism and application”. Reviews in Physics, 4: 100026, 2019.

[17] Stefan Fischer, Florian Hallermann, Toni Eichelkraut, Gero von Plessen, Karl W. Krämer, Daniel Biner, Heiko Steinkemper, Martin Hermle, and Jan C. Goldschmidt "Plasmon enhanced upconversion luminescence near gold nanoparticles-simulation and analysis of the interactions". Optics Express, 20 (1): 271, 2012.

[18] Yiming Wu, Jiahui Xu, Eng Tuan Poh, Liangliang Liang, Hailong Liu, Joel K. W. Yang, Cheng-Wei Qiu, Renaud A. L. Vallée, and Xiaogang Liu "Upconversion superburst with sub- $2 \mu$ s lifetime". Nature Nanotechnology, 14 (12): 1110-1115, 2019.

[19] Di M. Wu, Aitzol Garcia-Etxarri, Alberto Salleo, and Jennifer A. Dionne "Plasmon-Enhanced Upconversion". J. Phys. Chem. Lett., 5: 4020-4031, 2014.

[20] Angel Fernandez-Bravo, Danqing Wang, Ed- ward S. Barnard, Ayelet Teitelboim, Cheryl Tajon, Jun Guan, George C. Schatz, Bruce E. Cohen, Emory M. Chan, P. James Schuck, and Teri W. Odom "Ultralow-threshold, continuous- wave upconverting lasing from subwavelength plasmons". Nature Materials, 18: 1172-1176, 2019.

[21] Harry A. Atwater and Albert Polman "Plasmonics for improved photovoltaic devices". Nature Materials, 9 (3): 205213, 2010.

[22] Jiahui Xu, Zhaogang Dong, Mohamed Asbahi, Yim- ing Wu, Hao Wang, Liangliang Liang, Ray Jia Hong Ng, Hailong Liu, Renaud A. L. Vallée, Joel K. W. Yang, and Xiaogang Liu "Multiphoton Upconversion Enhanced by Deep Subwavelength Near-Field Confinement". Nano Letters, https://pubs.acs.org/action/showCitFormats?doi=10.1021/acs. nanolett.1c00232\&ref-pdf. 
[23] E. M. Purcell "Spontaneous Emission Probabilities at Radio Frequencies”. Physical Review, 69: 681, 1946.

[24] J. C. Goldschmidt, S. Fischer, P. Löper, K. W. Krämer, D. Biner, M. Hermle, and S. W. Glunz "Experimental analysis of upconversion with both coherent monochromatic irradiation and broad spectrum illumination". Solar Energy Materials and Solar Cells, 95 (7): 1960-1963, 2011.

[25] Prashant Nagpal, Nathan C. Lindquist, Sang-Hyun Oh, and David J. Norris "Ultrasmooth Patterned Met- als for Plasmonics and Metamaterials". Science, 325 (5940): 594 597, 2009.

[26] Zhaogang Dong, Mohamed Asbahi, Jian Lin, Di Zhu, Ying Min Wang, Kedar Hippalgaonkar, Hong-Son Chu, Wei Peng Goh, Fuke Wang, Zhiwei Huang, and Joel K. W. Yang
"Second-Harmonic Generation from Sub- $5 \mathrm{~nm}$ Gaps by Directed Self-Assembly of Nanoparticles onto TemplateStripped Gold Substrates”. Nano Letters, 15 (9): 5976-5981, 2015.

[27] Mohamed Asbahi, Shafigh Mehraeen, Fuke Wang, Nikolai Yakovlev, Karen S. L. Chong, Jianshu Cao, Mei Chee Tan, and Joel K. W. Yang "Large Area Directed Self-Assembly of Sub-10 nm Particles with Single Particle Positioning Resolution". Nano Letters, 15 (9): 6066-6070, 2015. Lumerical Inc.

[28] S. Fischer, H. Steinkemper, P. Löper, M. Hermle, and J. C. Goldschmidt "Modeling upconversion of erbium doped microcrystals based on experimentally determined Einstein coefficients". Journal of Applied Physics, 111 (1): 013109, 2012. 